\title{
Construct validity of perceptual style: Role of stimulus size in the embedded-figures test and the rod-and-frame test
}

\author{
MICHAEL J. STREIBEL \\ Instructional Systems Program, Division of Curriculum and Instruction \\ Pennsylvania State University, University Park, Pennsylvania 16802 \\ and \\ SHELDON M. EBENHOLTZ \\ Department of Psychology, University of Wisconsin, Madison, Wisconsin 53706
}

\begin{abstract}
Three experiments were performed in order to test the construct validity of perceptual field dependence (FD) and field independence (FI). In Experiment 1, performance of subjects on two sizes of Form A of Witkin's Embedded Figures Test (EFT) with a size-ratio of 1 to 4 was compared. The size of the standard EFT was taken as unity. A nonsignificant size effect was found for all subjects as a group, and a significant rank correlation was found between performances on the two sizes of the EFT. Furthermore, the performance of field-dependent subjects (as defined by performance on the standard EFT) and field-independent subjects did not interact significantly with the size factor. In Experiment 2, the physical size of the EFT changed from 1 to 8. A nonsignificant, overall size effect was again found, but the EFT performance of fielddependent and field-independent subjects interacted significantly with the size factor. In Experiment 3, the performance of subjects on two sizes of the EFT (i.e., 1 to 8) and two sizes of the standard rod-and-frame test (RFT) (i.e., 1 to 4.5) were compared. A significant stimulus-size effect was found in the EFT, with all subjects becoming more field-independent, and a significant stimulus-size effect was found in the RFT with the same subjects becoming more field-dependent. Finally, the EFT performance of the field-dependent and field-independent subjects again displayed a significant interaction with the size of the embedded figures. A parallel-serial processingstyles hypothesis is proposed to account for the EFT data.
\end{abstract}

It has been shown recently that changing the retinal size of a rod and frame (i.e., the angle that the rod and frame projects onto the retinal surface) modulates performance on the rod-and-frame test (RFT) such that the smaller the retinal angle, the lower the rod-and-frame effect (Ebenholtz, 1977; Ebenholtz \& Benzschawel, 1977). Since the RFT and the Embedded Figures Test (EFT) are both used as measures of perceptual style (Witkin, 1949; Witkin \& Asch, 1948; Witkin, Dyk, Faterson, Goodenough, \& Karp, 1962; Witkin, Lewis, Herzman, Machamer, Meissner, \& Wapner, 1954; Witkin, Oltman, Raskin, \& Karp, 1971), the question was raised as to whether the EFT would have the same pattern of stimulus-size effect as the RFT.

Witkin and his associates have long argued that the EFT and the RFT both measured the same thing. Perceptual field dependence/independence, or more generally, perceptual style, was defined (Witkin et al., 1971) as follows: "In a field-dependent mode of perceiving, perception is strongly dominated by the

This research was supported in part by N.I.H. Grant EY 02264 to S.M.E. overall organization of the surrounding field, and the parts are experienced as 'fused.' In a field-independent mode of perceiving, parts of the field are experienced as discreet from organized ground."

The claim that the EFT and the RFT measured the same thing has not been without its critics (Arbuthnot, 1972; Wachtel, 1972) and has usually been supported or denied on the basis of correlational studies (Long, 1974; Schiff, 1980). The present study investigated this claim within an experimental paradigm: If the EFT and the RFT tapped a common perceptual mechanism (i.e., perceptual style), then one would expect a similar shift in performance on both tests when a common stimulus variable such as size was manipulated.

\section{EXPERIMENT 1}

The first experiment was designed to test whether a stimulus-size effect existed in the Embedded Figures Test (EFT) when the size of the figures increased by a factor of four over the standard test. The experiment was also designed to test for a field-dependency and stimulus-size interaction when field de- 
pendency was measured by performance on the standard EFT.

\section{Method}

Apparatus. Two sizes of Form A of Witkin's EFT were constructed in a size ratio of 1 to 4. The "small" figures were constructed out of Coloraid paper and mounted on $38.1 \times 50.8 \mathrm{~cm}$ Crescent 300 illustration boards. Each of the "small" figures of Form A (i.e., 12 complex figures, 8 simple figures, and 2 practice figures) matched the figures of the original EFT produced by the Consulting Psychologists Press (Witkin et al., 1971) in color, shape, size, and orientation. Black lines in these figures were produced by using .04-cm-wide black matte Chartpak pressuresensitive graphic tape. The illustration boards were covered by $.191-\mathrm{cm}$ clear acetate and taped around the entire edges with Scotch Magic transparent tape. The "medium" figures of Form A were four times as large as the "small" figures, but were mounted on the same-size illustration boards.

The small and medium-sized figures were placed manually on a black, wooden, table-top easel, such that the center of each figure was $60.96 \mathrm{~cm}$ from, and perpendicular to, each subject's eye. The small figures therefore ranged in visual angle (i.e., the angle that the outer limits of the figures subtended on the retinal surface) from 2.4 to $6.2 \mathrm{deg}$ and the medium figures ranged from 9.6 to 24.8 deg. A $.32 \times 38.1 \times 50.8 \mathrm{~cm}$ clear Lexan sheet was placed on top of each figure so that a subject's manual tracing of the embedded figures would not scratch the original figures.

Procedure. The subject sat in an upright position with chin in a chinrest. The experimenter sat next to the subject on his or her right. The experimental room was dark except for a fluorescent lamp and an incandescent lamp, which were placed so that no light would shine directly into the subject's eyes. The test was administered according to the standard EFT instructions (Witkin et al., 1971), except that each subject saw six figures of the small EFT and six figures of the medium EFT (or vice versa) in the standard order instead of 12 figures of the same size.

Design. Eight male and nine female college students, between the ages of 18 and 32 years, from a number of introductory psychology classes at the University of Wisconsin were tested for their ability to disembed small and medium-sized EFT figures. They were given an experimental credit for their participation. These credits were part of a standard requirement for introductory psychology courses. The dependent variable reflected a speed and accuracy measure, and was calculated as the average response time for six small and six medium-sized figures of the EFT for each subject. Sex, stimulus size, sequence (i.e., small $\rightarrow$ medium vs. medium $\rightarrow$ small), order (i.e., first six figures vs. second six figures), and order within size (i.e., Figures 1-6 vs. 7-12 of Witkin's EFT) were counterbalanced, and the effects of these factors were calculated by a special ANOVA (Grant, 1949). One subject was dropped because she could not disembed the figures.

\section{Results}

Analyses of the EFT data from all subjects. A special ANOVA was calculated with these data (Grant, 1949), and a significant effect was not found for sequence $[F(1,14)=.14$, n.s.], order $[F(1,14)=.05$, n.s. $]$, or size $[F(1,14)=2.10, n$.s.]. The mean EFT response times for all subjects are given in Table 1.

Males were consistently faster than females, but separate one-way ANOVAs resulted in a nonsignificant sex effect [small EFT $F(1,14)=1.89$, n.s.; medium EFT $F(1,14)=2.71$, n.s.]

Finally, a significant Pearson correlation coefficient $[r=.694, p<.005]$ was found when the data from all subjects on the small and the medium EFT were compared. Subjects who were FD on the small
Table 1

Mean EFT Response Times (in Seconds) for All Subjects in Experiment 1

\begin{tabular}{lrcc} 
& \multicolumn{3}{c}{$\mathrm{RT}$} \\
\cline { 3 - 4 } Subjects & $\mathrm{n}$ & Mean & $\mathrm{SD}$ \\
\hline & & Small EFT \\
Total & 16 & 45.4 & 27.5 \\
Male & 8 & 36.2 & 27.8 \\
Female & 8 & 54.6 & 25.6 \\
& & Medium EFT & \\
Total & 16 & 37.4 & 26.5 \\
Male & 8 & 27.0 & 13.7 \\
Female & 8 & 47.7 & 32.8 \\
\hline
\end{tabular}

Note-Standard deviations of samples are based on $d f=n-1$.

Table 2

Mean EFT RTs for the Field-Independent (FI) and Field-Dependent (FD) Groups in Experiment 1

\begin{tabular}{cllll}
\hline $\begin{array}{c}\text { Perceptual } \\
\text { Style }\end{array}$ & $\begin{array}{c}\text { Size of } \\
\text { EFT }\end{array}$ & $\mathrm{n}$ & \multicolumn{2}{c}{ RT (in Seconds) } \\
\hline \multirow{2}{*}{ EFT FI } & Small & 5 & 15.5 & SD \\
& Medium & 5 & 26.3 & 10.7 \\
\multirow{2}{*}{ EFT FD } & Small & 5 & 78.0 & 15.0 \\
& Medium & 5 & 65.8 & 27.0 \\
\hline
\end{tabular}

Note-EFT field dependence (i.e., perceptual style) is defined by performance on the small EFT. Standard deviations of samples are based on $d f=n-1$.

EFT thus tended to be FD on the medium EFT. A Spearman rank correlation coefficient $\left[r_{s}=.5853\right.$, $t(15)=2.7, p<.01]$ confirmed this conclusion.

Analyses of the EFT data from field-dependent and field-independent subjects. Since there was not a significant sequence effect, the data from the two sequences were pooled and then ranked according to performance on the small EFT. The latter was done because the small EFT most closely approximated the size of Witkin's standard EFT. The 16 scores appeared normally distributed, and the top (i.e., fast response times) and bottom thirds (i.e., slow response times) were defined as field-independent and field-dependent, respectively. Table 2 summarizes the mean EFT data for these two groups. Although an ANOVA of data from the two groups showed a significant difference between field dependence and field independence $[F(1,8)=32.23, p<.01]$, neither a size effect $[F(1,8)=.02$, n.s. $]$ nor a field dependency $\times$ stimulus size interaction $[F(1,8)=3.41$, n.s. $]$ was found.

\section{Discussion}

The results of the first experiment indicated that performance in the EFT was not significantly affected by a fourfold increase in the size of the visual figures. The size of the standard Witkin EFT was taken as unity. Furthermore, a one-to-four size differential did not result in a significant interaction of the field-dependency and stimulus-size factors. 


\section{EXPERIMENT 2}

The second experiment was designed to test the effects of a one-to-eight figure-size differential on performance in the EFT. It was also designed to include a series of postsession questions about the size preferences and perceptual strategies of subjects.

\section{Method}

Apparatus. Two sizes of Form A of Witkin's EFT were constructed in a size ratio of 1 to 8 . The small EFT was identical to that used in Experiment 1. The stock of materials used to construct the "large" EFT was the same as that used for the figures in Experiment 1, except that the black lines of the large figures were made using .32-cm black matte Chartpak tape. The large figures, however, were mounted on $50.8 \times 67.5 \mathrm{~cm}$ illustration boards.

The table-top stand that supported the EFT figures was moved to a distance of $50.8 \mathrm{~cm}$ from each subject's eyes in order to amplify the size of the large figures. The small figures therefore ranged in visual angle from 2.6 to $6.6 \mathrm{deg}$, while the large figures ranged from 20.8 to 52.8 deg.

Procedure. All conditions in Experiment 2 were identical to those in Experiment 1, except that the subjects were asked a series of questions at the end of the EFT about their figure-size preferences and perceptual-processing strategies.

At the end of the EFT, the subjects were asked if they understood the nature of the perceptual disembedding task. If not, the purpose of the EFT was explained to them again. The subjects were then asked: "Did you find it easier to disembed the small or the large figures? By easy, I do not mean speed, but the degree to which you felt comfortable performing the task." Responses were recorded as either "small" or "large." The subjects were then asked: "What was your initial strategy for disembedding the simple figures? Did you first look for part of the simple figure within the larger complex figure and then look for other parts, or did you first look for the entire simple figure?" This question was designed to force a bimodal choice between a part-processing and a whole-processing perceptual strategy. If a subject persisted in saying that he or she had used both strategies, then his or her response was recorded as "part/whole."

Design. Twelve male and 14 female college students, between the ages of 18 and 25 and from a number of introductory psychology classes at the University of Wisconsin, were tested for their ability to disembed small and large figures. The experimental design was identical to that used in Experiment 1. Two female subjects were replaced because they displayed extremely large EFT response times relative to the other subjects (i.e., greater than three standard deviations above the group mean for one size of the EFT).

\section{Results}

Analyses of the EFT data from all subjects. An ANOVA of the data in Experiment 2 (Grant, 1949) did not show a significant sequence effect $[F(1,22)$ $=4.00$, n.s. $]$ or a significant size effect $[F(1,22)=.42$, n.s.], but did indicate a significant order effect $[F(1,22)=9.50, p<.05]$. The latter was attributed to practice. The mean EFT response times for all subjects are given in Table 3. As in Experiment 1, males were consistently significantly faster than females [small EFT $F(1,22)=9.78, p<.01$; large EFT $F(1,22)$ $=7.08, \mathrm{p}<.05]$.

A significant Pearson correlation coefficient $[\mathrm{r}=$ $.4111, \mathrm{p}<.01]$ was found when all the small EFT
Table 3

Mean EFT Response Times (in Seconds) for All Subjects in Experiment 2

\begin{tabular}{llcc} 
& \multicolumn{3}{c}{$\mathrm{RT}$} \\
Subjects & $\mathrm{n}$ & Mean & $\mathrm{SD}$ \\
\hline & \multicolumn{3}{c}{ Small EFT } \\
Total & 24 & 43.1 & 25.4 \\
Males & 12 & 29.3 & 19.0 \\
Females & 12 & 56.9 & 23.9 \\
& & Large EFT & \\
Total & 24 & 46.4 & 28.7 \\
Males & 12 & 32.6 & 20.0 \\
Females & 12 & 60.3 & 30.0 \\
\hline
\end{tabular}

Note-Standard deviations of samples are based on $d f=n-1$.

scores were compared with all the large EFT scores. A Spearman rank correlation calculation, however, failed to result in a significant correlation coefficient $\left[r_{s}=.3383\right.$, n.s.]. Ranking performance on the small EFT, therefore, could not be used to predict the rank of a subject's performance on the large EFT.

Analyses of the EFT data from field-dependent and field-independent subjects. The data from Experiment 2 was then ranked on the basis of performance on the small EFT. Table 4 summarizes the mean EFT scores of the field-dependent and fieldindependent subjects (i.e., eight subjects within each group). An ANOVA of the data from these two groups did not show a significant size effect $[F(1,14)$ $=1.45$, n.s.], but did show a significant field-dependency effect $[F(1,14)=8.24, p<.05]$ and a significant fielddependency $\times$ figure-size interaction $[F(1,14)=15.49$, $\mathrm{p}<.01]$.

Analyses of the size-preference and strategypreference data from the fleld-dependent and fleldindependent subjects. A Fisher exact nonparametric test indicated that the field-dependent and fieldindependent subjects did not differ significantly in the size of the figures they preferred to disembed $(p=.0609$ ]. A similar test of the disembedding-strategy data indicated that both groups looked primarily for parts of the embedded figures first $[p=.400]$.

\section{Discussion}

The results of the second experiment indicated that the small and large EFT (i.e., 1-to-8 size differential) could not be reliably considered to be interchangeable measures of the same thing. Although a significant size effect was not found for the performance of the entire group when the size of the embedded figures changed from 1 to 8 , a significant interaction between the field-dependency and the stimulus-size factors was found. Field-independent subjects (as defined by the standard, or small, EFT) became significantly more field-dependent, whereas field-dependent subjects became significantly more field-independent. The EFT performances of both 
Table 4

Mean EFT RTs for the Field-Independent (FI) and Field-Dependent (FD) Groups in Experiment 2

\begin{tabular}{|c|c|c|c|c|}
\hline \multirow{2}{*}{$\begin{array}{c}\text { Perceptual } \\
\text { Style }\end{array}$} & \multirow{2}{*}{$\begin{array}{c}\text { Size of } \\
\text { EFT }\end{array}$} & \multirow[b]{2}{*}{$\mathbf{n}$} & \multicolumn{2}{|c|}{ RT (in Seconds) } \\
\hline & & & Mean & SD \\
\hline EFT FI & $\begin{array}{l}\text { Small } \\
\text { Large }\end{array}$ & $\begin{array}{l}8 \\
8\end{array}$ & $\begin{array}{l}16.2 \\
45.8\end{array}$ & $\begin{array}{r}9.3 \\
31.4\end{array}$ \\
\hline EFT FD & $\begin{array}{l}\text { Smail } \\
\text { Large }\end{array}$ & $\begin{array}{l}8 \\
8\end{array}$ & $\begin{array}{l}70.2 \\
54.5\end{array}$ & $\begin{array}{l}18.1 \\
32.4\end{array}$ \\
\hline
\end{tabular}

Note-EFT field dependence (i.e., perceptual style) is defined by performance on the small EFT. Standard deviations of samples are based on $d f=n-1$.

groups, however, did not reach a common value. A nonsignificant rank correlation of the data from the entire group confirmed the conclusion that the two sizes of the EFT might not be measuring the same thing.

The disembedding-strategy data indicated that both groups looked primarily for parts of the embedded figures first. This was somewhat surprising, since the EFT is generally understood to be a gestaltbased perception test (Witkin et al., 1954). However, the strategy-preference data indicated that, although the EFT may have been designed according to gestalt principles (e.g., good form, continuity, symmetry, etc.), the processing styles of subjects did not necessarily proceed according to gestalt principles. Perceptual style may, in fact, tap how well a subject's serial processing of parts can overcome the distraction of natural gestalt groupings (more of this later).

\section{EXPERIMENT 3}

The third experiment was designed to replicate the second experiment and also expose all subjects to two sizes of the standard rod-and-frame test (RFT). Field dependence in the EFT was again measured by performance with the small figures, whereas field dependence in the RFT was measured by performance with the large rod and frame (Ebenholtz, 1977). The third experiment was therefore intended to go to the heart of the question of whether the EFT and RFT measure the same thing (i.e., perceptual field dependence/ independence): if a different pattern of performances by the same subjects was found in the EFT and the RFT, then a strong possibility would exist that these two tests did not measure the same thing. The latter conclusion would be especially strong if a different interaction pattern of the field-dependency and stimulus-size factors were to be found for the two tests.

\footnotetext{
Method

Apparatus. The two sizes of Form A of Witkin's EFT were identical to those used in Experiment 2, as were the procedures for administering and scoring the test. The RFT apparatus, however, was constructed specifically for this experiment.
}

The small and the large rod and frame consisted of a luminous line and a square outline-frame, respectively. They were painted on separate, black Crescent 648 illustration boards with Palmer's luminous paint. The dimensions of the small rod and frame were $8.5 \times .6 \mathrm{~cm}$ and $10.7 \times 10.7 \times .6 \mathrm{~cm}$, respectively. The dimensions of the large rod and frame were $40.1 \times .6 \mathrm{~cm}$ and $50.5 \times 50.5$ $\times .6 \mathrm{~cm}$, respectively. An identical line width was chosen for all figures so that the same light intensity would be radiated from any unit area. ${ }^{1}$

The plane of the rod-and-frame figures was situated perpendicularly to each subject's line of sight at a distance of $67.1 \mathrm{~cm}$. The small rod and frame therefore subtended a 9.1-deg visual angle, while the large rod and frame subtended a 41.3-deg visual angle. The small and large outline frames were permanently tilted $20 \mathrm{deg}$ clockwise when seen by the subject. The small and large rods were independently mounted and were rotated manually around the central axis, and in the same plane, of the frame. A large protractor in the rear part of the table-top apparatus permitted the degree of rod tilt to be measured with a .5-deg accuracy. Subjects initially fixated the center of the rod but were then free to view the entire figure throughout the experiment.

Before each RFT, the four RFT figures were simultaneously exposed to room light for $5 \mathrm{~min}$ in order to stimulate an equivalent amount of luminescent radiation. These figures were then stored out of the light in order to allow the brightest levels of luminescence to decay. The experimental room was totally dark during the RFT, and all surfaces in the room were painted with black, nongloss paint or covered with black, nonreflective cloth. The latter was done in order to eliminate even the faintest visual orientation cues (Irving \& Henderson, 1971; Long, 1973).

Procedure. Subjects adjusted the small and large rod to the apparent gravitational vertical from starting positions of 25 deg clockwise and 25 deg counterclockwise, with bracketing permitted (Ebenholtz, 1977). The rod-rotation rate was experimentercontrolled. The upright orientation of the rod was measured first in a no-frame condition and then within an appropriately sized frame condition. The frame, when present, was always tilted 20 deg clockwise. The rod-and-frame effect was defined as the algebraic difference (i.e., signed error) between the average of the two settings taken with the tilted-frame and no-frame conditions (Ebenholtz, 1977). ${ }^{2}$

RFT field-independence was defined as the upper third of the distribution of scores (i.e., the least deviation from the true gravitational upright), and RFT field dependence was defined as the lower third.

Design. Sixteen male and 18 female subjects were tested in all of the EFT and RFT conditions. Sixteen subjects were exposed first to the EFT and then to the RFT, while the remaining 16 were exposed to the reverse sequence. The EFT and RFT tests followed within minutes of each other. The design of the EFT was otherwise identical to that used in Experiment 2. Finally, two female subjects were replaced because they displayed extreme field dependence relative to other subjects (i.e., EFT scores greater than three standard deviations about the group mean).

The RFT consisted of eight trials with either a small-to-large or a large-to-small sequence. The four trials within any particular size consisted of a two-part rod-only condition (i.e., 25 deg clockwise and $25 \mathrm{deg}$ counterclockwise rod-starting position), followed by a two-part frame-present condition (i.e., 20 deg clockwise tilted frame with the same two rod starting positions). The RFT dependent variable for any particular sized frame was the difference between the average rod setting in degrees of the frame-present and the frame-absent conditions. The scores from the two rodstarting positions (i.e., $25 \mathrm{deg} \mathrm{cw}$ and $25 \mathrm{deg} \mathrm{ccw}$ ) were averaged in order to counterbalance any rod starting-position effects (Morant \& Arnoff, 1966; Werner \& Wapner, 1952). A positive RFT deviation indicated that the tilted frame modified the subject's perception of the upright in the direction of the frame. ${ }^{3}$ The rod, in such a case, was therefore set in the direction of the tilt of the frame in order to be aligned with the perceived upright. 
Results

Analyses of the EFT data from all subjects. An ANOVA of the EFT data in Experiment 3 (Grant, 1949) did not show a significant sequence effect $[F(1,30)=.53$, n.s.], but did show significant order $[F(1,30)=4.74, p<.05]$ and size $[F(1,30)=6.64$, $\mathrm{p}<.025]$ effects. The lack of a significant EFT sequence effect permitted the data from the two sequences to be pooled. The significant EFT order effect (i.e., first six figures vs. second six figures) was probably due to practice. The significant EFT size effect meant that all subjects as a group disembedded the large figures significantly faster than they disembedded the small figures. The mean EFT response times are given in Table 5 . Males were slightly slower than females, but this difference was not significant (small EFT $F(1,30)=.45$, n.s.; large EFT $\mathrm{F}(1,30)=.02$, n.s. $]$.

Analyses of the EFT data from field-dependent and field-independent subjects. The EFT data from Experiment 3 was ranked on the basis of performance on the small EFT, and the upper and lower thirds of the distribution were used to define field dependence/independence. Table 6 summarizes the mean EFT scores of the FD and FI subjects. An ANOVA of the data from these two groups revealed a significant size effect $[F(1,20)=23.15, p<.01]$, a significant difference between the FD and FI groups $[F(1,20)=19.42, p<.01]$, and a significant interaction between the field-dependency and stimulussize factors $[F(1,20)=47.97, p<.01]$. Further analyses showed that the performance of the FI and FD groups on the large $E F T$ did not differ significantly from each other $[t(21)=.14, n . s$.$] .$

Analyses of the size-preference and strategypreference data from field-dependent and fieldindependent subjects. A Fisher exact nonparametric test indicated a significant difference in the stated figure-size preferences $[p=.0062]$. Hence, FI subjects preferred to disembed small figures significantly more often than did FD subjects. The opposite was true for large figures. Since the size-preference ques-

Table 5

Mean EFT Response Times (in Seconds) for All Subjects in Experiment 3

\begin{tabular}{llcc} 
& \multicolumn{3}{c}{$\mathrm{RT}$} \\
\cline { 3 - 4 } Subjects & $\mathrm{n}$ & Mean & $\mathrm{SD}$ \\
\hline & & Small EFT \\
Total & 32 & 43.4 & 23.5 \\
Males & 16 & 46.4 & 25.9 \\
Females & 16 & 40.6 & 21.2 \\
& & Large EFT & \\
Total & 32 & 31.2 & 19.2 \\
Males & 16 & 31.7 & 21.1 \\
Females & 16 & 30.7 & 17.7 \\
\hline
\end{tabular}

Note-Standard deviations of samples are based on $d f=n-1$.
Table 6

Mean EFT RTs for the Field-Independent (FI) and Field-Dependent (FD) Groups in Experiment 3

\begin{tabular}{ccccc}
\hline & & & \multicolumn{2}{c}{ RT (in Seconds) } \\
\cline { 5 - 5 } Style & EFT & $\mathrm{n}$ & Mean & SD \\
\hline \multirow{2}{*}{ EFT FI } & Small & 11 & 20.3 & 8.7 \\
& Large & 11 & 27.8 & 17.8 \\
\multirow{2}{*}{ EFT FD } & Small & 11 & 70.0 & 15.5 \\
& Large & 11 & 28.3 & 18.9 \\
\hline
\end{tabular}

Note-EFT field dependence (i.e, perceptual style) is defined by performance on the small EFT. Standard deviations of samples are based on $d f=n-1$.

tion was asked in such a way as to avoid confusing size preference with speed, these results indicated that size preference in the EFT matched speedaccuracy performance. That is, FI subjects disembedded small figures relatively faster than they did large figures, and also preferred disembedding small figures. FD subjects disembedded large figures relatively faster than small figures and preferred disembedding large figures.

A Fisher exact nonparametric test of the initial search strategies indicated that FI subjects differed significantly from FD subjects $[p=.0386]$. FI subjects tended to look for the entire figure during the disembedding task (i.e., 8 of 11), whereas FD subjects tended to look for parts of the embedded figure (i.e., 8 of 11).

Analyses of the RFT data from all subjects. An ANOVA of the rod-and-frame-test (RFT) data (Grant, 1949) did not show a significant sequence effect $[F(1,30)=.66, n . s$.$] or a significant order effect [F(1,30)$ $=.04$, n.s.], but did result in a significant size effect $[F(1,30)=48.56, p<.001]$. This was consistent with earlier RFT research (Ebenholtz, 1977). The mean RFT deviations from the upright in degrees for the entire group are given in Table 7.

The mean RFT values in Table 7 reflect a rod-andframe effect based on signed errors (i.e., deviations from the true gravitational upright in the direction opposite to the frame tilt were coded as negative values). Only 5 of the 32 subjects displayed a negative rod-and-frame effect in response to the small frame, and none did so in response to the large frame. Hence, by using Witkin's definition of the RFT (i.e., based on unsigned errors), only the mean RFT values for the small frame changed, and they did so to a slight degree (see note to Table 7).

Analyses of the RFT data from field-dependent and field-independent subjects. Since there was no sequence effect, the RFT data were ranked according to performance on the large EFT. This was done because earlier research had shown that the large frames were better able to discriminate field-dependency (or perceptual style) than were small frames (Ebenholtz, 1977). Table 8 summarizes the mean 
Table 7

Mean RFT Scores (in Degrees) for All Subjects in Experiment 3

\begin{tabular}{llcc} 
& \multicolumn{2}{c}{$\mathrm{RT}$} \\
\cline { 3 - 4 } Subjects & $\mathrm{n}$ & Mean & $\mathrm{SD}$ \\
\hline & \multicolumn{3}{c}{ Small RFT } \\
Total & 32 & 1.50 & 1.54 \\
Males & 16 & 1.47 & 1.44 \\
Females & 16 & 1.53 & 1.67 \\
& & Large RFT & \\
Total & 32 & 4.98 & 2.60 \\
Males & 16 & 4.95 & 2.82 \\
Females & 16 & 5.02 & 2.53 \\
\hline
\end{tabular}

Note-Standard deviations of samples are based on $d f=n-1$. The mean RFT values for the small frame, when calculated according to Witkin's definition of the RFT (i.e., based on unsigned errors), yielded $1.72 \mathrm{deg}$, with a standard deviation of $1.28 \mathrm{deg}$ for the entire group. Males and females displayed a 1.63-deg and 1.81-deg rod-and-frame effect, respectively, with a slight drop in the magnitude of the standard deviation.

Table 8

Mean RFT Scores (in Degrees) for the Field-Independent (FI) and Field-Dependent (FD) Groups in Experiment 3

\begin{tabular}{ccccc}
\hline \multirow{2}{*}{$\begin{array}{c}\text { Perceptual } \\
\text { Style }\end{array}$} & $\begin{array}{c}\text { Size of } \\
\text { RFT }\end{array}$ & $\mathrm{n}$ & Mean & SD \\
\hline \multirow{2}{*}{ RFT FI } & Small & 11 & $.66^{*}$ & 1.06 \\
& Large & 11 & 2.59 & .80 \\
\multirow{2}{*}{ RFT FD } & Small & 11 & $1.52 \dagger$ & 1.27 \\
& Large & 11 & $\mathbf{8 . 0 0}$ & 1.95 \\
\hline
\end{tabular}

Note-RFT field dependence (i.e., perceptual style) is defined by performance on the large RFT. Standard deviations of samples are based on $d f=n-1$. Using unsigned errors to calculate the mean RFT for the small-frame condition yielded a value of .93 deg with a .80-deg standard deviation. †Using unsigned errors to calculate the mean RFT for the small-frame condition yielded a value of 1.75 deg with a .89-deg standard deviation.

Table 9

Pearson Correlation Matrices of the EFT and Signed RFT Data in Experiment 3

\begin{tabular}{cccr}
\hline \multicolumn{1}{c}{ Test } & Large EFT & Small RFT & Large RFT \\
\hline & \multicolumn{3}{c}{ (a) Total Group $(\mathrm{n}=32)$} \\
Small EFT & .1231 & -.0291 & -.0416 \\
Large EFT & & -.0828 & .0766 \\
Small RFT & & & .1917 \\
& & (b) Males $(\mathrm{n}=16)$ & \\
Small EFT & .2252 & .2258 & .0347 \\
Large EFT & & -.0758 & .1753 \\
Small RFT & & & -.0322 \\
& & (c) Females (n = 16) & \\
Small EFT & -.0307 & -.2931 & -.1426 \\
Large EFT & & -.0913 & -.0532 \\
Small RFT & & & .4088 \\
\hline
\end{tabular}

Note-Each of these correlation coefficients failed to reach significance (i.e., $\alpha<.05$ ).
RFT scores for the FI and FD subjects (i.e., the upper and lower thirds of the distribution -11 subjects within each group).

The mean RFT values in Table 8 again reflected the signed errors of RFT performance. Since the values from the large frame condition were used to determine field dependency, and since none of these values were negative, the issue of signed vs. unsigned errors became a moot point. However, a slight change in the mean RFT scores for the field-dependent and field-independent subjects in the small-frame condition can be seen when the unsigned errors are taken into account (see notes to Table 8). An ANOVA of these data (i.e., signed error) resulted in a significant size effect $[F(1,20)=116.36, p<.001]$, a significant difference between FI and FD subjects $[F(1,20)=$ $58.76, p<.001]$, and a significant interaction between the field-dependency and frame-size factors $[F(1,20)=33.41, p<.001]$. The interaction effect was the result of FD subjects' becoming more field dependent at a faster rate than FI subjects as the size of the stimuli got larger.

An ANOVA of the unsigned RFT data from the FD and FI subjects in Experiment 3 indicated the same conclusions. The size effect $[F(1,20)=129.17$, $p<.001]$, the field dependency effect $[F(1,20)=$ $69.72, \mathrm{p}<.001$ ], and the field-dependency and framesize interaction $[F(1,20)=43.34, p<.001]$ were all significant.

Correlational analyses of the EFT and RFT data from all subjects. A Pearson correlation coefficient matrix of the entire data set from Experiment 3 did not result in any significant correlations (see Table 9). The same correlations were recalculated using the unsigned errors in the RFT (see Table 10). Hence, the possibility existed that all four tests (i.e., small and large EFT and RFT) measured independent perceptual processes. Since the small-large RFT correlations came close to significance for the entire group, a Spearman rank correlation was performed on all the data (see Table 11). This time, the small-large RFT correlations reached significance for the entire group $\left[r_{s}=.3222, p<.05\right]$, which, in turn, was attributed to the female RFT performance $\left[r_{s}=.4757\right.$, $\mathrm{p}<.05]$.

A further test of all the data was made in order to see if any other associations could be found. A $2 \times 2$ Fisher exact nonparametric test based on a median split resulted only in a significant association between the small and the large RFT [p=.0055]. All other measures indicated that the performances were made by independent groups even though each person took part in all conditions.

Analyses of the EFT and RFT data from FI and FD subjects within each test. It was hypothesized that, perhaps, if performance on the large RFT were used as a general definition of FI and FD, then one would have a better predictor of performance on the 
Table 10

Pearson Correlation Matrices of the EFT and Unsigned RFT Data in Experiment 3

\begin{tabular}{cccc}
\hline \multicolumn{1}{c}{ Test } & Large EFT & Small RFT & Large RFT \\
\hline & \multicolumn{3}{c}{ (a) Total Group $(\mathrm{n}=32)$} \\
Small EFT & .1231 & -.0981 & -.0416 \\
Large EFT & & -.0710 & .0766 \\
Small RFT & & .2219 \\
& & (b) Males $(\mathrm{n}=16)$ \\
Small EFT & .2253 & .1733 & .0347 \\
Large EFT & & -.1846 & .1753 \\
Small RFT & & & .1427 \\
& & (c) Females $(\mathrm{n}=16)$ \\
Small EFT & -.0307 & -.3910 & -.1426 \\
Large EFT & & .0573 & -.0532 \\
Small RFT & & & .3052 \\
\hline
\end{tabular}

Note-Each of these correlation coefficients failed to reach significance (i.e., $\alpha<.05$ ).

Table 11

Spearman Rank-Correlation Matrices of the EFT and Signed RFT Data in Experiment 3

\begin{tabular}{cccc}
\hline \multicolumn{1}{c}{ Test } & Large EFT & Small RFT & Large RFT \\
\hline & \multicolumn{3}{c}{ (a) Total Group $(\mathrm{n}=32)$} \\
Small EFT & .0695 & -.0415 & -.1478 \\
Large EFT & & -.0455 & .1010 \\
Small RFT & & & $.3222^{*}$ \\
& & (b) Males $(\mathrm{n}=16)$ & \\
Small EFT & .2118 & .2537 & -.0059 \\
Large EFT & & -.0944 & .1990 \\
Small RFT & & & .1234 \\
& & (c) Females $(\mathrm{n}=16)$ \\
Small EFT & -.1324 & -.3299 & -.2356 \\
Large EFT & & -.0707 & .0074 \\
Small RFT & & & $.4757^{*}$ \\
\hline
\end{tabular}

${ }^{*} p<.05 ;$ all others were nonsignificant.

EFT. Accordingly, the EFT scores of subjects defined FI and FD on the RFT were examined. An ANOVA indicated that field dependency in the RFT could not be used as a predictor of field dependency in the EFT $[F(1,20)=.10$, n.s.]. Furthermore, the interaction of the field-dependency and stimulus-size factors in the RFT did not show up for the same subjects in the EFT. Nonsignificant Pearson correlations and Spearman rank correlations confirmed this lack of association.

The converse of the previous argument was also examined: If performance on the small EFT were used as a general definition of FI and FD, could one predict performance in the RFT? Accordingly, the RFT scores of the FI and FD subjects were examined. An ANOVA indicated that grouping subjects on the EFT did not distinguish these subjects as FI or FD in the RFT $[F(1,20)=.22$, n.s. $]$.

Analyses of the small EFT data from all subjects in Experiments 1-3. A one-way ANOVA of the small EFT data from all subjects across all experiments showed that the mean performance levels on the small EFT did not differ significantly from each other $[F(2,69)=.04, n . s$. $]$. The three small EFTs were identical to each other and matched the Witkin EFT in figure size, shape, color, and orientation. The oneway analysis of the small EFT data therefore indicated that, since the three samples of subjects used in the three experiments came from the same population, the EFT analyses could be compared across experiments (see General Discussion below).

\section{Discussion}

The third experiment indicated a number of differences between the small and large EFT and between the EFT and RFT. First, the small and large EFT (i.e., 1-to-8 size differential) could not be considered interchangeable measures of the same thing because there existed a significant stimulus-size effect and because FD and FI subjects (as measured on the small EFT) could not be distinguished from each other on the large EFT. More importantly, a significant interaction between the field-dependency and stimulus-size factors was again found. FD and FI subjects, in effect, were differentially affected by an increase in the size of the embedded figures.

The third experiment also indicated that the EFT and the RFT might not be measuring the same thing. In the EFT, all subjects became more field-dependent as the size of the embedded figures increased from 1 to 8 . In the RFT, the same subjects became more field-independent as the size of the rod and frame increased from 1 to about 4.5. The average visual angle of the small and large EFT figures ranged from about 5 to 40 deg, respectively, whereas the visual angle of the small and large rods and frames ranged from 9 to about $41 \mathrm{deg}$.

The interaction patterns of the field-dependency and stimulus-size factors (as defined within each test) also differed between the EFT and the RFT. This was most evident for FD subjects. In the EFT, FD subjects became significantly more field-independent as the size of the stimuli increased, whereas, in the RFT, FD subjects became more field-dependent. If the two tests had measured a common perceptual mechanism called field-independence (or more generally, perceptual style), then the field-dependency and stimulus-size interaction patterns should not have differed.

An attempt was also made to see if the performance of the FD and FI subjects in the EFT could predict field dependence/independence of the same subjects in the RFT, and vice versa. In neither case could the field-dependence factor, or the interaction between the field-dependency and the stimulus-size factors, be predicted from one test to the other test. This conclusion was confirmed by the correlation matrices that were performed when the scores from all the subjects were taken into account.

The size-preference data in Experiment 3 confirmed the implications of Experiment 2: FI subjects 
preferred to disembed small figures significantly more often than did FD subjects; FD subjects preferred to disembed large figures. The strategy-preference data indicated that FI subjects looked for the entire figures during the disembedding task, whereas FD subjects looked for parts of the intended figures. Could these results be related? That is, could it be that preferring to disembed small figures is somehow related to looking for entire gestalts and preferring to disembed large figures is related to looking for parts of the intended figures? Also, could these preferences and strategies account for the interaction between the field-dependency and stimulus-size factors in the EFT? A parallel-serial processing-styles hypothesis will be presented later to accomplish just that.

One final note about Experiment 3. The small and large RFTs were the only pair of tests that showed any degree of statistical association. It was therefore concluded that the small and large RFT measured the same thing in spite of the fact that a stimulus-size effect and a field-dependency and stimulus-size interaction were found. A brief glance at the data from FD and FI subjects in the RFT shows that all the subjects became significantly more field-dependent as the size of the stimuli got large. The small and large RFT discriminated between the same subjects. In the EFT, on the other hand, performances of FD and FI subjects moved in opposite directions as the size of the stimuli got larger (see Figures 1 and 2).

\section{GENERAL DISCUSSION}

The three experiments described thus far indicated that the performance of FD and FI subjects in the EFT was differentially sensitive to the size of the embedded figures. This sensitivity was significant only when the embedded figures were eight times as large as the standard size. In Experiment 1 (i.e., 1-4 EFT size differential), the field-dependency and stimulus-size factors did not interact, but they did interact significantly in Experiments 2 and 3 (i.e., 1-8 EFT size differential). Figure 1 shows the mean EFT scores for the FD and FI subjects from all three experiments. The results from the three experiments can be compared with each other because the performances of the three groups of subjects on the standard (or "small") EFT did not differ significantly from each other.

The effect of stimulus size in the EFT on all subjects, however, was not as clear. First of all, stimulus size affected the performances of FD and FI subjects in opposite manners: FD subjects became significantly more field-independent when the size of the figures increased by a factor of eight, and FI subjects became significantly more field-dependent. The effect of stimulus size therefore tended to be masked when the scores of all subjects were averaged

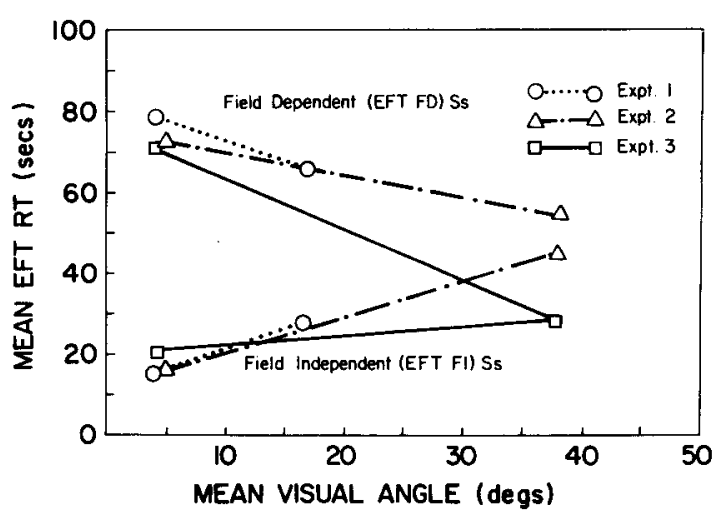

Figure 1. Embedded Figures Test (EFT) means for EFT FI and FD subjects in Experiments 1-3.

together. Second, Experiments 2 and 3 gave somewhat conflicting results, inasmuch as the performances of all subjects remained the same in Experiment 2 and became more field-independent in Experiment 3 when the size of the figures increased (see Tables 1, 3, 5).

We can gain an understanding of the effect of stimulus-size on all the subjects if we examine the correlation coefficients from the three experiments. Witkin has reported a significant test-retest correlation of $r=.89$ for the EFT (Witkin et al., 1971). This corresponds to a one-to-one (1-1) size differential for the standard EFT. Experiment 1 (i.e., 1-4 size differential) resulted in a significant correlation of $r=.694$, whereas Experiments 2 and 3 (i.e., 1-8 size differential) resulted in both a significant correlation of $r=$ .411 and a nonsignificant correlation of $r=.1231$, respectively. Thus, the tendency of increasing figure size is to decrease the correlation of the performances between the standard and the larger size EFT.

What kind of hypotheses could account for these data? Witkin and his colleagues' definition of perceptual style suggests that field dependence is a stable psychological characteristic which, although subject to a learning effect, remains fairly constant throughout adulthood (Witkin et al., 1971). If this definition were true, how could FD subjects become significantly more field-independent as the size of the EFT increased from 1 to 8 ? In fact, in Experiment 3, the "large" EFT (i.e., 8 times as large as the standard EFT) failed to discriminate between these two groups of subjects! Witkin's definition is supported somewhat by the evidence provided by FI subjects in Experiment 3 , since their performance remained relatively invariant with respect to stimulus size. However, the performance of FI subjects in Experiment 2 (which was identical to Experiment 3) varied to a significant degree.

The size-preference and strategy-preference data may provide us with a possible hypothesis. In Experi- 
ment 3, FI subjects tended to prefer the small stimulus figures, and FD subjects tended to prefer the large figures. Great care was taken in establishing these preference data to avoid a confusion between speed of EFT performance and size preference. Therefore, perhaps there was something in the way in which FI subjects processed the small figures that differed from the way in which FD subjects processed the large figures (i.e., other than speed of performance). The clue for such a difference came from the strategypreference data. The subjects were asked, after the EFT, whether they had looked for one part of the simple figures and then for other parts until they had identified the entire simple figure, or, whether they had initially looked for the entire simple figure. The former was labeled "serial processing"' (i.e., searching for parts in a linear fashion and trying to identify the simple figure sequentially), and the latter was labeled "parallel processing" (i.e., searching for the entire figure at once and treating it as a gestalt).

One-way ANOVAs of the strategy-preference data of FD and FI subjects in Experiment 2 did not show a significant strategy-preference effect, whereas the same analysis in Experiment 3 did show a significant strategy preference effect for the small figures $[F(2,29)$ $=3.4, p<.05]$. Subjects in Experiment 3 who did well on the small EFT (i.e., fast and accurate response times) also tended to be those subjects who reported using whole-figure search strategies (i.e., parallel processing style). Subjects who were slow to disembed the small figures tended to be those subjects who reported using part-figure search strategies (i.e., serial processing style). Furthermore, when the data from those subjects in Experiment 3 who said that they had used both strategies were examined, 8 of 10 said they had searched: (1) for the entire simple figure while disembedding the small figures, and (2) for the parts of the simple figure while disembedding the large figures.

The final component of the parallel-serial processing styles hypothesis was provided by an a priori argument in response to the question, "Why did the EFT performance of FI and FD subjects differ on the standard EFT?" A proposed answer was that parallel processing (i.e., preferred by the FI subjects) was inherently faster than serial processing (i.e., preferred by the FD subjects). Furthermore, the size of the large figures militated against a subject's perceiving the entire simple figure as a gestalt (i.e., the visual angle of the largest figure was $52 \mathrm{deg}$ ), while the size of the smallest figure permitted gestalt perception (i.e., the visual angle of the smallest figure was $2.2 \mathrm{deg}$ ).

The question then became, "Why did the performances of FD and FI subjects shift in opposite directions when the size of the embedded figures increased?" The proposed parallel-serial processing styles hypotheses can answer this question as follows:

(1) Small figures (i.e., visual angle between 2.2 and $6.6 \mathrm{deg}$ ) place a parallel processing demand on a subject in a disembedding task, whereas large figures (i.e., visual angle between 20.80 and $52.8 \mathrm{deg}$ ) place a serial-processing demand on a subject.

(2) Some subjects prefer to disembed visual figures in a parallel manner and are therefore fast when the task demands such a processing style (i.e., with small figures). They get increasingly slower as the stimulus size gets larger because the task begins to demand more and more of a serial processing style.

(3) Other subjects prefer to disembed visual figures in a serial manner and are therefore fast when the task demands such a processing style (i.e., with large figures). They get increasingly slower as the stimulus size gets smaller because the task begins to demand more of a parallel processing style.

(4) The performance differences of FI and FD subjects on the standard (or "small") EFT can be attributed to the presumption that parallel processing (i.e., whole-figure processing) is inherently faster than serial processing.

It should be noted that the parallel-serial processing styles hypothesis presented above is a composite statement which attempts to be internally as well as externally consistent with all the data. The data from the three experiments were only partially consistent with Witkin's concept of perceptual field dependence/independence.

Witkin believed that FI subjects were more "analytical" than FD subjects because they could "break up" a stimulus field into parts and ignore the peripheral parts. He also believed that field dependence/ independence was a stable psychological characteristic throughout adulthood. Accordingly, the performance of FI subjects should remain constant as the size of the embedded figures increases. This was found to be the case in Experiment 3. If we add the a priori argument that a stimulus pattern naturally breaks into parts as the stimulus gets larger, then FI subjects should become faster as the figures get larger. This was not found.

FD subjects, according to Witkin's hypothesis, ought also to perform at a constant EFT level as the size of the embedded figures increases. The data, however, indicated that FD subjects became faster as the size of the stimuli got larger. We could attribute this to the presumption that large visual gestalts perceptually break up of their own accord.

The second major finding of these experiments was that the Embedded Figures Test (EFT) and the rod-andframe test (RFT) might not have measured a common variable called field dependence/independence, or, more generally, perceptual style. Here again, this conclusion was indicated by the correlational calculations of the data from all subjects and the field- 


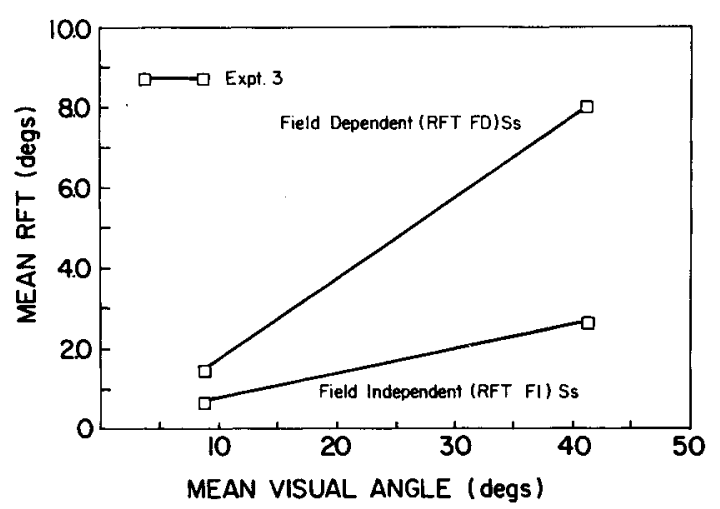

Figure 2. Rod-and-frame-test (RFT) means for RFT FI and FD subjects in Experiment 3.

dependence $x$ stimulus-size interaction data from FD and FI subjects. Even though all subjects in Experiment 3 were exposed to all sizes of the EFT and RFT in a completely counterbalanced design, only their performances on the small and the large RFT correlated to any significant degree. All other correlations were low and nonsignificant. This conclusion was supported by the finding that the performance of FD and FI subjects as defined by one test was not distinguishable to any significant degree from that defined by the other test. Finally, the interaction patterns of the field-dependency and stimulus-size factors differed for the same subjects on the two tests (see Figures 1 and 2). Most significant were the performance changes of FD subjects. In the EFT, FD subjects became significantly more field-independent, whereas in the RFT, FD subjects became significantly more field-dependent. If the two tests tapped a common mechanism, then the performance of subjects most susceptible to that mechanism should not have changed in opposite directions as the size of the stimuli increased by a factor of eight. The stimulus-size effect in the RFT has been tentatively explained by the "two visual systems" hypothesis (Ebenholtz, 1977):

The present experiments therefore suggest that the two sizes of the EFT (i.e., 1-8 ratio) might not be tapping a common perceptual mechanism called field dependence/independence (or perceptual style), and that the EFT and the RFT are not interchangeable measures of perceptual style. Researchers need to be wary of using the EFT and the RFT interchangeably, and they need to control for the size of the EFT in future perceptual styles research. Finally, educational researchers who use the EFT as a measure of perceptual style will have to be sure that the size of their visual stimuli in a learning task matches the size of the visual figures in the EFT (Ausburn \& Ausburn, 1978). The results of Experiment 3 also indicated that the EFT does not discriminate field dependence/ independence when the mean visual angle of visual figures is about $37 \mathrm{deg}$.

\section{REFERENCES}

Arbuthnot, J. Cautionary note on measurement of field dependence. Perceptual and Motor Skills, 1972, 35, 479-488.

Ausburn, L. J., \& Ausburn, F. B. Cognitive styles: Some information and implications for instructional design. Educational Communication and Technology Journal, 1978, 26, 337-354.

Emenholtz, S. M. Determinants of the rod and frame effect: The role of retinal size. Perception \& Psychophysics, 1977, 22, 531-538.

Ebenholtz, S. M., \& Benzschawel, T. L. The rod and frame effect and induced head tilt as a function of observation distance. Perception \& Psychophysics, 1977, 22, 491-496.

Grant, D. W. The statistical analysis of a frequent experimental design. American Journal of Psychology, 1949, 62, 119-122.

Inving, D., \& Henderson, D. On the validity of the portable rod-and-frame test. Perceptual and Motor Skills, 1971, 32, 434.

Leibowitz, H. W., \& Post, R. B. Two modes of processing concept and some implications, 1981. In J. Beck (Ed.), Organization and representation in perception. Hillsdale, N.J: Erlbaum, in press.

Long, G. M. The rod-and-frame test: Further comments on methodology. Perceptual and Motor Skills, 1973, 36, 624-626.

LoNG, G. M. Reported correlates of perceptual style: A review of the field-dependency-independency dimension. APA Journal Supplement No. 540, 1974, 1-82.

Morant, R. B., \& ARnoff, J. Starting position, adaptation, and visual framework as influencing the perception of verticality. Journal of Experimental Psychology, 1966, 71, 684-688.

Schiff, W. Perception: An applied approach. Boston: Houghton Mifflin, 1980.

WAChtel, P. L. Field dependence and psychological differentiation: Reexamination. Perceptual and Motor Skills, 1972, 35, 179-189.

Wenderoth, P. M. The distinction between the rod-and-frame illusion and the rod-and-frame test. Perception \& Psychophysics, $1974,19,205-212$.

Werneh, H., \& WAPNER, S. Experiments on sensory-tonic field theory of perception: IV. Effect of initial position of a rod on apparent verticality. Journal of Experimental Psychology, 1952, 43, 68-74.

Witkin, H. A. Perception of body position and of the position of the visual field. Psychological Monographs: General and Applied, 1949, 63, No. 302.

WitK IN, H. A., \& Asch, S. E. Studies in space orientation: IV. Further experiments on perception of the upright with displaced visual fields. Journal of Experimental Psychology, 1948, 38, 762-782.

Witkin, H. A., Dyk, R. B., Faterson, H. F., Goodenough, D. R., \& KARP, S. A. Psychological differentiation: Studies of development. New York: Wiley, 1962.

Witkin, H. A., Lewis, H. B., Herzman, M., Machamer, K., Meissner, P. B., \& Wapnen, S. Personality through perception: An experimental and clinical study. New York: Harper, 1954.

Witkin, H. A., Oltman, P. K., Raskin, E., \& Karp, S. A. $A$ manual for the Embedded Figures Test. Palo Alto, Calif: Consulting Psychologists Press, 1971.

\section{NOTES}

1. The different surface areas of the two rods and frames are a potential confound in the present study. A stimulus surface-area 
effect has been found in a periphery of the retina for induced circular vection (Leibowitz \& Post, 1981).

2. The rod-and-frame effect was also calculated by averaging the unsigned error in order to see if Witkin's original definition of the RFT covaried with stimulus size (Witkin, 1949; Witkin \& Asch, 1948). More of this later.

3. The use of a signed error as a measure of the RFT in this paper meant that the authors chose to relate the rod-and-frame effect to a measure of the mean. The present authors therefore believed that the directionality, as well as the magnitude, of the RFT measure was an important component of the rod-and-frame effect. Witkin, by using only the unsigned error, stressed the importance of the variance of the RFT measure in the rod-andframe effect (Wenderoth, 1974).

(Manuscript received May 2, 1981; revision accepted for publication October 13, 1981.) 